\title{
Adventures in diffraction - from atomic form factors to the resolution of defects
}

\author{
Peter. G. Khalifah ${ }^{a, b^{*}}$, Liang Yin ${ }^{a, b}$, Gerard Matteia,b, and Zhuo Li $i^{a, b}$ \\ ${ }^{a}$ Department of Chemistry, Stony Brook University, USA \\ ${ }^{b}$ Department of Chemistry, Brookhaven National Laboratory, USA \\ *kpete@bnl.gov
}

\begin{abstract}
Although the Rietveld refinement of powder diffraction data from synchrotron and neutron sources can in theory be used to solve and refine structural models with great accuracy, in practice there are many challenges that often prevent these goals from being achieved. The development of modern computational tools offers many new opportunities for solving hard problems in experimental crystallography. Some recent explorations of crystallographic frontiers will be discussed, potentially including (1) using powder diffraction to identify and resolve problems associated with the standard neutral form factors in complex oxides taking advantage of a new approach for representing diffraction parameter space that can be used to sensitively probe occupancy defects, (2) the use of structuregenerating algorithms as a tool for structure solution, and (3) the Rietveld refinement of $\sim 10^{5}$ diffraction patterns collected in operando to enable the battery devices to be followed with high spatial and temporal sensitivity.
\end{abstract}

